\title{
Quasi-Optimal Triangulations for Gradient Nonconforming Interpolates of Piecewise Regular Functions
}

\author{
A. Agouzal * and N. Debit \\ University Lyon1, Institute Camille Jordan, UMR 5208, 69100 Villeurbanne, France
}

\begin{abstract}
Anisotropic adaptive methods based on a metric related to the Hessian of the solution are considered. We propose a metric targeted to the minimization of interpolation error gradient for a nonconforming linear finite element approximation of a given piecewise regular function on a polyhedral domain $\Omega$ of $\mathbb{R}^{d}, d \geq 2$. We also present an algorithm generating a sequence of asymptotically quasi-optimal meshes relative to such a nonconforming discretization and give numerical asymptotic behavior of the error reduction produced by the generated mesh.
\end{abstract}

Key words: finite elements, anisotropic meshes

AMS subject classification:65D05, 65D15, 65N50

\section{Introduction}

Adaptive methods are essential for the successful simulation of various problems modelling natural phenomena occurring in geoscience, biology and hydrogeology... due to the anisotropic nature of the solution involved. This anisotropy make it necessary to consider both size and stretching during adaption. Metric Based Adaptivity ([1], [3] and references therein), where mesh adaption is carried out by optimization rather than by re-meshing, is independent of the specific problem being solved and provides stretched meshes to capture boundary and internal layers. The motivation of such metric based methods came from interpolation estimates. This work reports on a recent contribution to MBA methods for simplicial meshes in case of nonconforming finite element approximation.

*Corresponding author :E-mail:agouzal@univ-lyon1.fr 


\section{On quasi-optimal meshes and error gradient reduction}

Let $\Omega$ be a polyhedral connected domain of $\mathbb{R}^{d}(d=2,3)$ and $I\left(u, \triangle_{N}(\Omega)\right)$ denoting the linear nonconforming interpolate of a given piecewise regular function $u$ at the edges (faces) of the conformal triangulation $\triangle_{N}(\Omega)$ with at most $N$ d-simplex. We set

$$
\forall \Delta \in \triangle_{N}(\Omega), \quad I_{\Delta} u=I\left(u, \triangle_{N}(\Omega)\right),
$$

and denote by $\nabla_{h} I\left(u, \triangle_{N}(\Omega)\right)$ the vector field defined by

$$
\forall \Delta \in \triangle_{N}(\Omega), \quad \nabla_{h} I\left(u, \triangle_{N}(\Omega)\right)=\nabla I\left(u, \triangle_{N}(\Omega)\right) \text { on } \Delta .
$$

In the sequel, for any d-simplex $\Delta$ with vertices $\left(a_{i}\right)_{i=1}^{d+1},|\Delta|$ denotes the volume of $\Delta$ in the Euclidean metric, and for any symmetric positive definite matrix $G$, we denote the total length of $\Delta$ and volume of $\Delta$ in the metric $G$ respectively by

$$
|\partial \Delta|_{G}=\sum_{1 \leq i<j \leq d+1}\left\|G\left(a_{i}-a_{j}\right)\right\| \quad \text { and } \quad|\Delta|_{G}=\int_{\Delta} \sqrt{\operatorname{det} G} d x
$$

where $\|\cdot\|$ stands hereafter for the Euclidean norm.

The first result deals with the lower asymptotic behavior of the nonconforming interpolation gradient error.

Theorem 1. Let $u$ be a $C^{2}$-piecewise function and $H$ its Hessian. We have:

$$
\forall p \in] 0, \infty], \quad \liminf _{N \longrightarrow+\infty} N^{\frac{1}{d}} \inf _{\triangle_{N}(\Omega)}\left\|\nabla u-\nabla_{h} I\left(u, \triangle_{N}(\Omega)\right)\right\|_{L^{p}(\Omega)} \geq C(d, p)\left(\int_{\Omega} \sqrt{\mathcal{M}_{p}} d x\right)^{\frac{d+p}{p d}}
$$

where metric $\mathcal{M}_{p}$ is defined by

$$
\mathcal{M}_{p}=\left(\operatorname{det} H^{2}\right)^{-\frac{1}{d+p}} H^{2}
$$

and the constant

$$
C(d, p)= \begin{cases}\frac{(d !)^{\frac{1}{d}} d^{\frac{1}{2}}(d+1)^{-\frac{d+1}{2 d}}}{\sqrt{d+2}}, & \text { if } p \geq 2, \\ \frac{(d !)^{\frac{1}{d}} d^{\frac{1}{2}}(d+1)^{-\frac{d+1}{2 d}}}{((d+1)(d+2))^{\frac{1}{p}}}, & \text { if } p<2,\end{cases}
$$

the infimum being taken over all triangulations with $N$-simplex.

To prove Theorem 1, we need the following auxiliary results. The first one gives a geometric property of the involved metric: 
Lemma 2. For any $\Delta$ a d-simplex with vertices $\left(a_{i}\right), i=1, \ldots, d+1$, and $u$ a quadratic function on $\Delta$, we have

$$
\forall p \geq 1, \quad(d !)^{\frac{1}{d}} d^{\frac{1}{2}}(d+1)^{-\frac{d+1}{2 d}} \times|\Delta|_{H^{2}}^{\frac{1}{d}} \leq\left(\frac{1}{d+1} \sum_{i=1}^{d+1}\left\|H\left(a_{i}-x_{\Delta}\right)\right\|^{p}\right)^{\frac{1}{p}},
$$

where $x_{\Delta}$ is the barycenter of $\Delta$ and $H$ the Hessian of $u$, and the equality holds for $H^{2}$-regular $d$-simplex $\Delta$ if $\operatorname{det} H \neq 0$.

The following lemma concerns an integral representation of the gradient interpolation error based on the fundamental integration theorem,

Lemma 3. Let $\Delta$ be a d-simplex and $u \in \mathcal{C}^{2}(\bar{\Delta})$, we have

$$
\forall x \in \Delta, \quad \nabla u(x)-\nabla I_{\Delta} u(x)=\frac{1}{|\Delta|} \int_{\Delta} \int_{0}^{1} H(y+t(x-y)) .(x-y) d y d t,
$$

where $H$ is the Hessian of $u$.

Proof. Let $e(x)=u(x)-I_{\Delta} u(x)$ be the interpolation error. First we have for $x, y \in \Delta$,

$$
\nabla e(x)=\nabla e(y)+\int_{0}^{1} H(y+t(x-y)) \cdot(x-y) d t .
$$

Integrating with respect to $y$ over $\Delta$ and using the fact that $\int_{\Delta} \nabla e(y) d y=0$, prove the assertion of the lemma.

Let us remark in case $u \in P_{2}(\Delta)$, we obtain the equality

$$
\nabla u(x)-\nabla I_{\Delta} u(x)=H\left(x-x_{\Delta}\right),
$$

where $x_{\Delta}$ is the barycenter of $\Delta$.

Finally, using this last remark and lemma 2 gives a geometric representation of the gradient interpolation error:

Lemma 4. For any $\Delta$ a d-simplex with vertices $\left(a_{i}\right), i=1, \ldots, d+1$, and $u$ a quadratic function on $\Delta$, we have:

$$
(d !)^{\frac{1}{d}} d^{\frac{1}{2}}(d+1)^{-\frac{d+1}{2 d}}|\Delta|_{H^{2}}^{\frac{1}{d}} \leq\left\|\nabla u-\nabla I_{\Delta} u\right\|_{L^{\infty}(\Delta)}=\sup _{i=1, \ldots, d+1}\left\|H\left(a_{i}-x_{\Delta}\right)\right\|,
$$

and

$$
\frac{(d !)^{\frac{1}{d}} d^{\frac{1}{2}}(d+1)^{-\frac{d+1}{2 d}}}{\sqrt{d+2}}|\Delta|^{\frac{1}{2}}|\Delta|_{H^{2}}^{\frac{1}{d}} \leq\left\|\nabla u-\nabla I_{\Delta} u\right\|_{L^{2}(\Delta)}=\frac{|\Delta|^{\frac{1}{2}}}{\sqrt{d+2}}\left(\frac{1}{(d+1)} \sum_{i}\left\|H\left(a_{i}-x_{\Delta}\right)\right\|^{2}\right)^{\frac{1}{2}},
$$

where $x_{\Delta}$ is the barycenter of $\Delta$ and $H$ the Hessian of $u$, and the equality holds for $H^{2}$-regular $d$-simplex $\Delta$. 
Let us notice that, in general case, for any given quadratic function $u$ and d-simplex $\Delta$, the following inequalities on interpolation error hold:

1. For any $p \geq 2$, we have:

$$
\begin{aligned}
|\Delta|^{\frac{1}{p}-\frac{1}{2}}\left(\int_{\Delta}\left(u-I_{\Delta} u\right)^{2} d x\right)^{\frac{1}{2}} \leq\left(\int_{\Delta}\left(u-I_{\Delta} u\right)^{p} d x\right)^{\frac{1}{p}} \leq & \left\|\left(u-I_{\Delta} u\right)\right\|_{L^{\infty}(\Delta)}^{1-\frac{2}{p}} \times \\
& \left(\int_{\Delta}\left(u-I_{\Delta} u\right)^{2} d x\right)^{\frac{1}{p}} .
\end{aligned}
$$

2. For any $p, 0<p<2$,

$$
\begin{array}{r}
\left\|\left(u-I_{\Delta} u\right)\right\|_{L^{\infty}(\Delta)}^{1-\frac{2}{p}} \times\left(\int_{\Delta}\left(u-I_{\Delta} u\right)^{2} d x\right)^{\frac{1}{p}} \leq\left(\int_{\Delta}\left(u-I_{\Delta} u\right)^{p} d x\right)^{\frac{1}{p}} \leq|\Delta|^{\frac{1}{p}-\frac{1}{2}} \times \\
\left(\int_{\Delta}\left(u-I_{\Delta} u\right)^{2} d x\right)^{\frac{1}{2}} .
\end{array}
$$

These results are crucial to derive the following lemma, final step to prove Theorem 1.

Lemma 5. For any $\Delta a d$-simplex with vertices $\left(a_{i}\right), i=1 \ldots d+1$, and u a quadratic function on $\Delta$, then, for $p \geq 2$, we have:

$$
\begin{aligned}
\frac{(d !)^{\frac{1}{d}} d^{\frac{1}{2}}(d+1)^{-\frac{d+1}{2 d}}}{\sqrt{d+2}}|\Delta|_{H_{\Delta, p}}^{\frac{d+p}{d \times p}} \leq\left\|\nabla u-\nabla I_{\Delta} u\right\|_{L^{p}(\Delta)} \leq & \frac{(d+1)^{\frac{1}{2}-\frac{1}{p}}}{(d+2)^{\frac{1}{p}}}|\Delta|_{H_{\Delta, p}}^{\frac{1}{p}} \times \\
& \left(\frac{1}{d+1} \sum_{i=1}^{d+1}\left\|H\left(a_{i}-x_{\Delta}\right)\right\|^{2}\right)^{\frac{1}{2}},
\end{aligned}
$$

and for $0<p<2$, we have:

$$
\begin{aligned}
\frac{(d !)^{\frac{1}{d}} d^{\frac{1}{2}}(d+1)^{-\frac{d+1}{2 d}}}{((d+1)(d+2))^{\frac{1}{p}}}|\Delta|_{H_{\Delta, p}}^{\frac{d+p}{d \times p}} \leq\left\|\nabla u-\nabla I_{\Delta} u\right\|_{L^{p}(\Delta)} \leq \frac{1}{\sqrt{d+2}}|\Delta|_{H_{\Delta, p}}^{\frac{1}{p}} \times \\
\left(\frac{1}{d+1} \sum_{i=1}^{d+1}\left\|H\left(a_{i}-x_{\Delta}\right)\right\|^{2}\right)^{\frac{1}{2}},
\end{aligned}
$$

where $x_{\Delta}$ is the barycenter of $\Delta, H$ the Hessian of $u$ and metric $H_{\Delta, p}=(\operatorname{det} H)^{-\frac{2}{d+p}} H^{2}$. 
The second result of this paper deals with quasi-optimal metric-based meshes. Following the algorithm described in ([1], [3]) we generate, for a given piecewise regular function $u$, a sequence $\triangle_{N}(\Omega)$ of asymptotically quasi-optimal triangulations of the domain $\Omega$ in the case of linear nonconforming interpolation. More precisely, we have:

Theorem 6. Let $u$ be a $C^{2}$ - piecewise function on $\Omega$ and $H$ its Hessian, $\left.p \in\right] 0,+\infty[$ and $\Delta(N)$ a conformal mesh of $\Omega$ with at most $N d$-simplex. We assume that there exist a piecewise metric $G$ and positive constants $\sigma_{0}, \sigma_{1}$ such that:

1. $\forall \Delta \in \Delta(N), \forall(x, \zeta) \in \Delta \times \mathbb{R}^{d}, \quad\|H(x) \zeta\| \leq\|G \zeta\|$,

2. $\forall \Delta \in \Delta(N),|\partial \Delta|_{G} \leq \sigma_{0}|\Delta|_{G}^{\frac{1}{d}}$,

3. $N \times \sup _{\Delta \in \Delta(N)}|\Delta|_{G} \leq \sigma_{1}|\Omega|_{\mathcal{M}_{p}}$.

Then

$$
\left\|\nabla u-\nabla_{h} I\left(u, \triangle_{N}(\Omega)\right)\right\|_{L^{p}(\Omega)} \leq \frac{\sigma_{0} \sigma_{1}^{\frac{1}{p}+\frac{1}{d}}}{N^{\frac{1}{d}}}\left(\int_{\Omega} \sqrt{\mathcal{M}_{p}} d x\right)^{\frac{d+p}{p d}},
$$

where $\mathcal{M}_{p}$ is the piecewise metric defined by

$$
\forall \Delta \in \Delta(N), \quad \mathcal{M}_{p}=(\operatorname{det} G)^{-\frac{2}{d+p}} G^{2} .
$$

Remark 7. Technical steps of the proofs have been skipped and are not included in the paper for more readability. They will be given in an extended forthcoming paper.

\section{Numerical example}

To illustrate the good behavior of the generated mesh, we consider the problem of minimizing the interpolation error gradient of the analytical 'benchmark' function proposed in [2] over the domain $[0,1]^{2}$,

$$
u(x, y)=\frac{(x-0.5)^{2}-(\sqrt{10} y+0.2)^{2}}{\left((x-0.5)^{2}+(\sqrt{10} y+0.2)^{2}\right)^{2}},
$$

with weak anisotropic singularity in $(0.5,-0.2 / \sqrt{10})$, outside the computational domain but close to its boundary. 

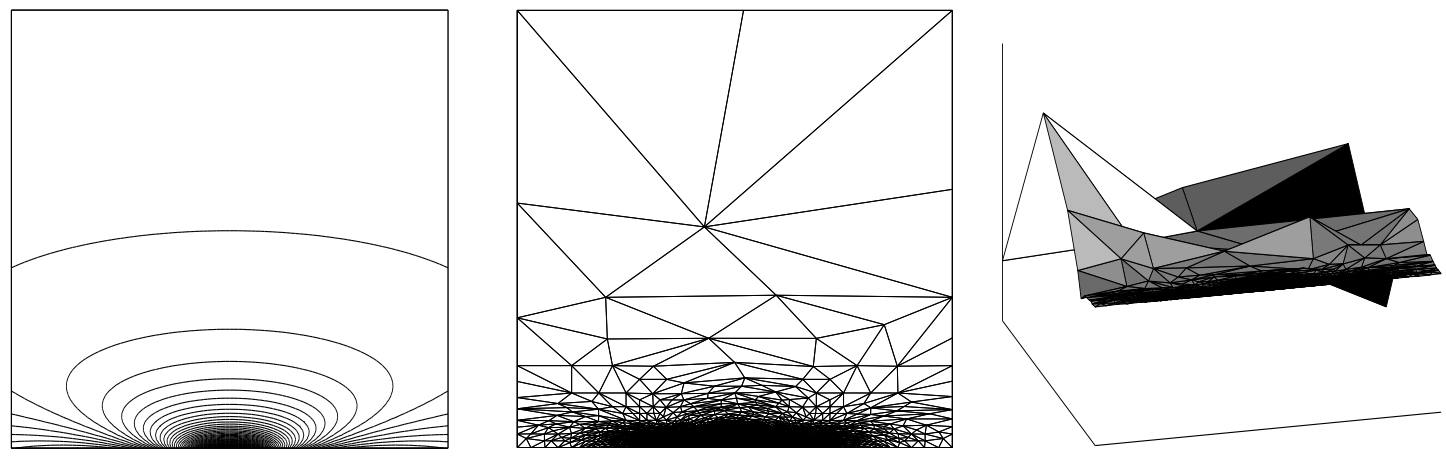

Figure 1: The isolines of the function $u(x, y)=\frac{(x-0.5)^{2}-(\sqrt{10} y+0.2)^{2}}{\left((x-0.5)^{2}+(\sqrt{10} y+0.2)^{2}\right)^{2}}$ (left); the mesh minimizing the $L_{\infty}$-norm of error gradient (middle) and its distribution on the computational domain (right).

\section{Acknowledgement}

These numerical simulations have been carried out using the software package Ani2D developed by Konstantin Lipnikov and Yuri Vassilevski: http://sourceforge.net/projects/ani2d/.

\section{References}

[1] A. Agouzal, K. Lipnikov, Y. Vassilevski. Generation of quasi-optimal meshes based on a posteriori error estimates. Proceedings of 16th International Meshing Roundtable. M.Brewerxi and D.Marcum (eds.), Springer, (2007), 139-148.

[2] E. D'Azevedo. Optimal triangular mesh generation by coordinate transformation. SIAM J. Sci. Comput., 12 (1991), 755-786.

[3] Y. Vassilevski, K. Lipnikov. Adaptive algorithm for generation of quasi-optimal meshes. Comp. Math. Math. Phys.,39 (1999), 1532-1551. 